\title{
Tilted axis cranking covariant density functional theory and its applications
}

\author{
P. W. Zhao ${ }^{1, \text { a }}$, H. Z. Liang ${ }^{1}$, J. Meng ${ }^{1,2,3}$, J. Peng ${ }^{4}$, P. Ring ${ }^{5,1}$, L. F. Yu ${ }^{1}$, and S. Q. Zhang ${ }^{1}$ \\ ${ }^{1}$ State Key Lab Nucl. Phys. \& Tech., School of Physics, Peking University, Beijing 100871, China \\ ${ }^{2}$ School of Physics and Nuclear Energy, Beihang University, Beijing 100191, China \\ ${ }^{3}$ Department of Physics, University of Stellenbosch, Stellenbosch, South Africa \\ ${ }^{4}$ Department of Physics, Beijing Normal University, Beijing 100875, China \\ ${ }^{5}$ Physik Department, Technische Universitat Muenchen, D-85747 Garching, Germany
}

\begin{abstract}
Recent progress on tilted axis cranking covariant density functional theory and its applications to nuclear magnetic and antimagnetic rotation are briefly presented. In particular, the magnetic rotation band in ${ }^{198} \mathrm{~Pb}$ and the antimagnetic rotational band in ${ }^{105} \mathrm{Cd}$ are discussed.
\end{abstract}

\section{Introduction}

Covariant density functional theory (CDFT) with a few number of parameters allows a very successful description of both ground state and excited state properties of nuclei all over the nuclide chart. In this report, we will mainly focus on our recent works on tilted axis cranking CDFT and its application to the microscopic investigation of magnetic rotation (MR) [1] and antimagnetic rotation (AMR) [2, 3].

It is quite common that rotational bands in nuclei are built on states with substantial quadrupole deformations. Over the past decades, however, the magnetic rotation bands have been discovered experimentally in nearly spherical nuclei with strong $M 1$ and weak $E 2$ transitions [4-6]. In a magnetic rotor, the magnetic moment is the order parameter inducing a violation of rotational symmetry, which forms an analogy to a ferromagnet where the total magnetic moment, the sum of the atomic dipole moments, is the order parameter. Since the first clear evidence for magnetic rotation observed in ${ }^{198,199} \mathrm{~Pb}$, up to date, more than 195 magnetic dipole bands spread over 85 nuclides have been observed [7].

In analogy with an antiferromagnet, on the other hand, a similar phenomenon is predicted in nuclei known as "antimagnetic rotation" [5]. The AMR differs from magnetic rotation distinctly. Firstly, the $M 1$ transitions in the AMR band are zero since the transverse magnetic moment vanishes. Secondly, as the antimagnetic rotor is symmetric with respect to a rotation by $\pi$ about the angular momentum axis, the spin difference of neighboring levels in AMR band is $2 \hbar$. Moreover, the AMR is characterized by a decrease of the $B(E 2)$ values with spin, which has been demonstrated by lifetime measurements [8]. To date, experimental evidences for AMR have been reported mainly in the Cd isotopes [8-13]. Other candidates include ${ }^{100} \mathrm{Pd}[14],{ }^{112} \mathrm{In}[15]$, and ${ }^{144} \mathrm{Dy}[16]$.

ae-mail: pwzhao@pku.edu.cn

This is an Open Access article distributed under the terms of the Creative Commons Attribution License 2.0, which permits unrestricted use, distribution, and reproduction in any medium, provided the original work is properly cited. 
From the theoretical side, both the MR and AMR bands have been discussed in simple geometry [6] and the cranking model [17] so far. The cranking model can explicitly construct the classical angular momentum vector which is of great help to understand the structure of rotational bands. In particular, based on the tilted axis cranking (TAC) approximation, lots of applications are carried out in the framework of the pairing plus quadrupole model (PQTAC) [5, 17]. Recently, cranked shell model with a particle-number-conserving approach [18] has also been applied to investigate the AMR [19].

However, in order to take the polarization effects fully into account and to treat the nuclear currents self-consistently, one should resort to fully self-consistent and microscopic investigations based on reliable theories without additional parameters. The framework of CDFT [20-22] is a good choice of such calculations. The CDFT was initially combined with the aplanar TAC model in the selfconsistent cranking relativistic mean-field (RMF) framework [23]. However, because of its numerical complexity, so far, it has been applied only for the magnetic rotation in ${ }^{84} \mathrm{Rb}$. Focusing on the magnetic rotation, a completely new computer code for the self-consistent TAC-RMF theory has been established [24]. Very recently, the TAC model based on a relativistic point-coupling Lagrangian which allows considerable simplification was established and applied successfully to the magnetic rotation in both light nuclei such as ${ }^{60} \mathrm{Ni}$ [25], ${ }^{58} \mathrm{Fe}$ [26] and heavy nuclei such as ${ }^{198,199} \mathrm{~Pb}$ [1]. Moreover, it provides the first fully self-consistent and microscopic investigation for the AMR band in ${ }^{105} \mathrm{Cd}[2,3]$. For more details on tilted axis cranking CDFT, one can refer to a recent review in Ref. [7].

In the present contribution, the microscopic CDFT investigation for the MR and AMR will be presented and the details can be found in Refs. [1-3].

\section{Theoretical Framework}

The starting point of CDFT with a point-coupling interaction is an effective Lagrangian density including a free-nucleon term, four-fermion point-coupling terms, higher order terms accounting for the medium effects, the derivative terms to simulate the finite-range effects, and the electromagnetic interaction terms. The details of this Lagrangian density can be seen in Refs. [27-29].

In the TAC model based on this point-coupling Lagrangian, it is assumed that the nucleus rotates around an axis in the $x$ - $z$ plane and the Lagrangian is transformed into a frame rotating uniformly with a constant rotational frequency,

$$
\mathbf{\Omega}=\left(\Omega_{x}, 0, \Omega_{z}\right)=\left(\Omega \cos \theta_{\Omega}, 0, \Omega \sin \theta_{\Omega}\right),
$$

where $\theta_{\Omega}$ is the tilted angle. One can derive the equations of motion for the nucleons

$$
[\boldsymbol{\alpha} \cdot(-i \boldsymbol{\nabla}-\boldsymbol{V})+\beta(m+S)+V-\boldsymbol{\Omega} \cdot \hat{\boldsymbol{J}}] \psi_{k}=\epsilon_{k} \psi_{k},
$$

where $\hat{\boldsymbol{J}}$ is the total angular momentum, and the relativistic fields $S(\boldsymbol{r}), V^{\mu}(\boldsymbol{r})$ read

$$
\begin{aligned}
S(\boldsymbol{r}) & =\alpha_{S} \rho_{S}+\beta_{S} \rho_{S}^{2}+\gamma_{S} \rho_{S}^{3}+\delta_{S} \Delta \rho_{S}, \\
V^{\mu}(\boldsymbol{r}) & =\alpha_{V} j_{V}^{\mu}+\gamma_{V}\left(j_{V}^{\mu}\right)^{3}+\delta_{V} \Delta j_{V}^{\mu}+\tau_{3} \alpha_{T V} j_{T V}^{\mu}+\tau_{3} \delta_{T V} \triangle j_{T V}^{\mu}+e A^{\mu},
\end{aligned}
$$

with $e$ the electric charge unit vanishing for neutrons. Here, the time components of $j_{V}^{\mu}$ and $j_{T V}^{\mu}$ correspond to the vector density $\rho_{V}$ and the isovector-vector density $\rho_{T V}$, respectively. The total energy in the laboratory frame reads

$$
E_{\mathrm{tot}}=E_{\mathrm{kin}}+E_{\mathrm{int}}+E_{\mathrm{cou}}+E_{\mathrm{cm}},
$$

which is composed of a kinetic part $E_{\text {kin }}$, an interaction part $E_{\text {int }}$, an electromagnetic part $E_{\text {cou }}$, and the center-of-mass correction energy $E_{\mathrm{cm}}$. Following the standard procedure, one can also obtain the expectation values of the angular momenta, the quadrupole moments, and the electromagnetic transition probabilities. The detailed formalism and numerical techniques can be seen in Refs. [3, 24]. 


\section{Results and Discussion}
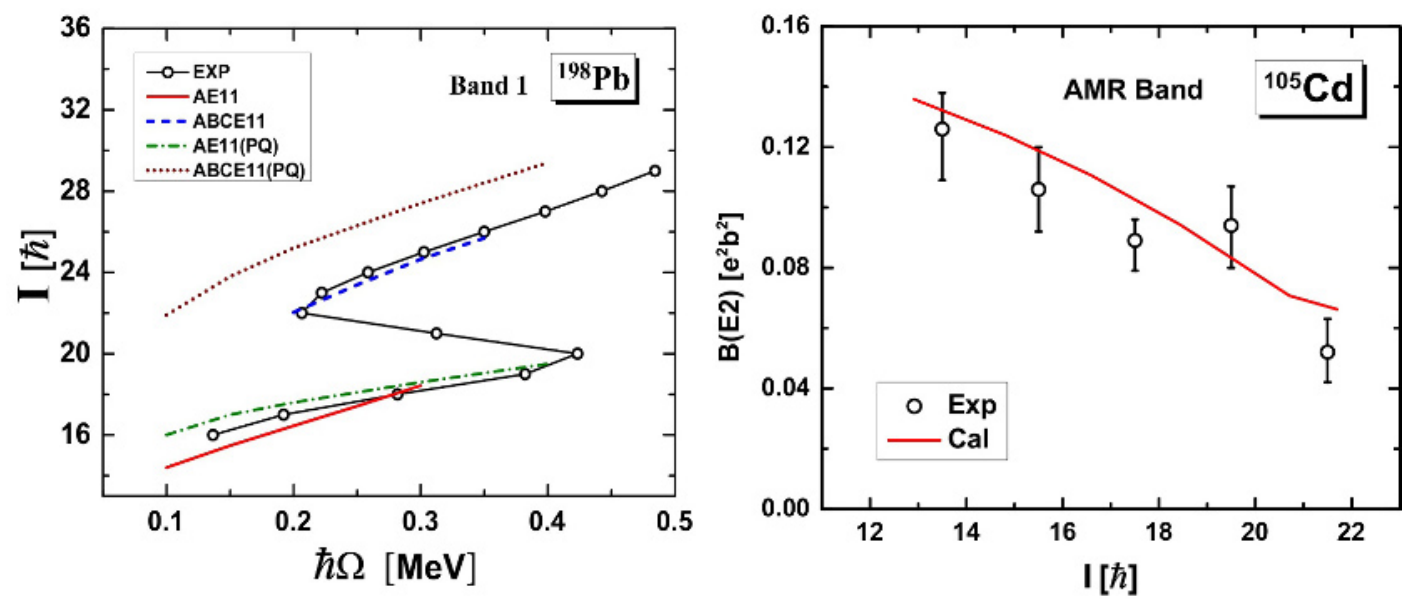

Figure 1. (Color online) Left: Angular momenta as a function of the rotational frequency in the tilted axis cranking CDFT calculations compared with the data [30] and the PQTAC results [31] for Band 1 in ${ }^{198} \mathrm{~Pb}$. Taken from Ref. [1]. Right: $B(E 2)$ values as a function of the angular momentum in the tilted axis cranking CDFT calculations compared with data [9] for the AMR band in ${ }^{105} \mathrm{Cd}$. Taken from Ref. [2].

In the left panel of Fig. 1, the calculated total angular momenta for one of the MR bands, Band 1, in ${ }^{198} \mathrm{~Pb}$ as a function of the rotational frequency are shown in comparison with the data [30] and the PQTAC results [31]. It is found that both the tilted axis cranking CDFT and the PQTAC results agree well with the experimental data. Here, as in Ref. [1], the proton configuration $\pi\left[s_{1 / 2}^{-2} h_{9 / 2} i_{13 / 2}\right] 11^{-}$is abbreviated by its spin number 11 , and the neutron configurations $v\left[i_{13 / 2}^{-1}(f p)^{-1}\right]$ is referred as AE11 before the backbending, and $v\left[i_{13 / 2}^{-3}(f p)^{-1}\right]$ as ABCE11 after the backbending. The TAC calculations support that the backbending arises through an excitation of a neutron-hole pair in the $i_{13 / 2}$ shell. After the backbending, the PQTAC results are nearly $3 \hbar$ larger than the data and the CDFT results.

Antimagnetic rotation is characterized by weak $E 2$ transitions decreasing with spin. In the right panel of Fig. 1, the calculated $B(E 2)$ values are compared with the available data for the AMR band in ${ }^{105} \mathrm{Cd}$ [9]. The resulting $B(E 2)$ values are very small $\left(<0.14 e^{2} b^{2}\right)$ and in very good agreement with the data. Furthermore, the fact that the $B(E 2)$ values decrease with spin is in agreement with the interpretation by the two shearslike mechanism. The decrease of the $B(E 2)$ values can also be understood by the change of deformation. Actually, as discussed in Ref. [2], the nucleus undergoes a rapid decrease of $\beta$ deformation from 0.2 to 0.14 at a small and near-constant triaxiality. Therefore, one can conclude that the alignment of the proton and neutron angular momenta, i.e., the two shearslike mechanism, is accompanied by a transition from prolate towards nearly spherical shape.

\section{Summary}

In summary, the self-consistent tilted axis cranking covariant density functional theory with a pointcoupling interaction and its application to the magnetic rotation and antimagnetic rotation in nuclei are briefly reported. Without any additional parameter, the experimental relation between angular momentum and rotational frequency for the magnetic rotation band in ${ }^{198} \mathrm{~Pb}$ as well as the data of $B(E 2)$ values for the antimagnetic rotation band in ${ }^{105} \mathrm{Cd}$ are reproduced very well. 


\section{Acknowledgments}

This work was supported in part by the Major State 973 Program of China (Grant No. 2013CB834400), the Natural Science Foundation of China (Grants No. 11175002, No. 11105005, No. 11105006), the China Postdoctoral Science Foundation (Grant No. 2012M520101, No. 2013T60022), and the Oversea Distinguished Professor Project from Ministry of Education No. MS2010BJDX001. We also acknowledge partial support from the DFG Cluster of Excellence "Origin and Structure of the Universe"(www.universe-cluster.de).

\section{References}

[1] L.F. Yu, P.W. Zhao, S.Q. Zhang, P. Ring, J. Meng, Phys. Rev. C 85, 024318 (2012)

[2] P.W. Zhao, J. Peng, H.Z. Liang, P. Ring, J. Meng, Phys. Rev. Lett. 107, 122501 (2011)

[3] P.W. Zhao, J. Peng, H.Z. Liang, P. Ring, J. Meng, Phys. Rev. C 85, 054310 (2012)

[4] H. Hübel, Prog. Part. Nucl. Phys. 54, 1 (2005)

[5] S. Frauendorf, Rev. Mod. Phys. 73, 463 (2001)

[6] R.M. Clark, A.O. Macchiavelli, Annu. Rev. Nucl. Part. Sci. 50, 1 (2000)

[7] J. Meng, J. Peng, S.Q. Zhang, P.W. Zhao, Front. of Phys. 8, 55 (2013)

[8] A.J. Simons et al., Phys. Rev. Lett. 91, 162501 (2003)

[9] D. Choudhury et al., Phys. Rev. C 82, 061308 (2010)

[10] A.J. Simons et al., Phys. Rev. C 72, 024318 (2005)

[11] P. Datta et al., Phys. Rev. C 71, 041305 (2005)

[12] S. Roy et al., Phys. Lett. B 694, 322 (2011)

[13] C.J. Chiara et al., Phys. Rev. C 61, 034318 (2000)

[14] S. Zhu et al., Phys. Rev. C 64, 041302 (2001)

[15] X.W. Li et al., Phys. Rev. C 86, 057305 (2012)

[16] M. Sugawara et al., Phys. Rev. C 79, 064321 (2009)

[17] S. Frauendorf, Nucl. Phys. A 677, 115 (2000)

[18] J. Zeng, T. Cheng, Nucl. Phys. A 405, 1 (1983)

[19] Z.H. Zhang, P.W. Zhao, J. Meng, J.Y. Zeng, E.G. Zhao, S.G. Zhou, Phys. Rev. C 87, 054314 (2013)

[20] P. Ring, Prog. Part. Nucl. Phys. 37, 193 (1996)

[21] D. Vretenar, A.V. Afanasjev, G.A. Lalazissis, P. Ring, Phys. Rep. 409, 101 (2005)

[22] J. Meng, H. Toki, S. Zhou, S. Zhang, W. Long, L. Geng, Prog. Part. Nucl. Phys. 57, 470 (2006)

[23] H. Madokoro, J. Meng, M. Matsuzaki, S. Yamaji, Phys. Rev. C 62, 061301 (2000)

[24] J. Peng, J. Meng, P. Ring, S.Q. Zhang, Phys. Rev. C 78, 024313 (2008)

[25] P.W. Zhao, S.Q. Zhang, J. Peng, H.Z. Liang, P. Ring, J. Meng, Phys. Lett. B 699, 181 (2011)

[26] D. Steppenbeck et al., Phys. Rev. C 85, 044316 (2012)

[27] B.A. Nikolaus, T. Hoch, D.G. Madland, Phys. Rev. C 46, 1757 (1992)

[28] T. Bürvenich, D.G. Madland, J.A. Maruhn, P.G. Reinhard, Phys. Rev. C 65, 044308 (2002)

[29] P.W. Zhao, Z.P. Li, J.M. Yao, J. Meng, Phys. Rev. C 82, 054319 (2010)

[30] A. Görgen et al., Nucl. Phys. A 683, 108 (2001)

[31] S. Chmel, S. Frauendorf, H. Hübel, Phys. Rev. C 75, 044309 (2007) 